\title{
The Effects of Nano Fillers on Space Charge Distribution in Cross-Linked Polyethylene
}

\author{
A. N. Ramani ${ }^{1}$, A. M. Ariffin ${ }^{2}$, Gobinath Vijian ${ }^{3}$, and Ahmad Basri Abd Ghani ${ }^{4}$ \\ ${ }^{1}$ Faculty of Electrical Engineering, Universiti Teknikal Malaysia Melaka, Malaysia \\ ${ }^{2,3}$ Department of Electrical Power Engineering, College of Engineering, Universiti Tenaga Nasional, Malaysia \\ ${ }^{4}$ TNB Research Sdn. Bhd., Malaysia
}

\section{Article Info}

\section{Article history:}

Received: Jun 6, 2017

Revised: Aug 21, 2017

Accepted: Sep 3, 2017

\section{Keyword:}

Nanocomposites

Nanodielectrics

Polyethylene

Space charge

PEA

\begin{abstract}
The performance of polymeric insulation will be distorted by the accumulation of space charge. This will lead to local electric field enhancement within the insulation material that can cause degradation and electrical breakdown. The introduction of nanofillers in the insulation material is expected to reduce the space charge effect. However, there is a need to analyze potential nanofillers to determine the best option. Therefore, the objective of this research work is to examine two types of nanofillers for Cross-Linked Polyethylene (XLPE); Zinc Oxide ( $\mathrm{ZnO}$ ) and Acrylic (PA40). The effects of these nanofillers were measured using the Pulsed-Electro Acoustic (PEA) method. The development of space charge is observed at three different DC voltage levels in room temperature. The results show that hetero charge distribution is dominant in pure XLPE materials. The use of both nanofiller types have significant effect in decreasing the space charge accumulation. With nanofillers, the charge profile changed to homo-charge distribution, suppressing the space charge formation. Comparison between both the nanofillers show that PA40 has better suppression performance than $\mathrm{ZnO}$.
\end{abstract}

Copyright (C) 2017 Institute of Advanced Engineering and Science. All rights reserved.

\author{
Corresponding Author: \\ Anis Niza Ramani \\ Universiti Teknikal Malaysia Melaka \\ Hang Tuah Jaya, 76100 Durian Tunggal, Melaka \\ Emailanisniza@utem.edu.my
}

\section{INTRODUCTION}

Polymer materials are widely used in many electrical application due to appropriate processing ability and excellent electrical insulation properties. Examples include polyethylene (PE), polypropylene (PP), polyimide (PI) and etc. However, there is still a need to further improve the insulation properties of the materials. Therefore, there are many studies that focused on the development of the materials using the concept of composite in order to improve material properties [1]. Recently, research works have been concentrating on the application of nanomaterial to enhance the insulation properties.

A lot of work has been done already on conduction current measurement and space charge observation, mostly several decades ago regarding conduction current and in the last 10 years for space charge [2]. The presence of space charge modifies the electric field, increasing the internal field locally within the dielectric material, which leads to faster degradation and premature failure of the material [3]. The mechanism of space charge formation is therefore considered as one of the most influential factors in determining the overall dielectric properties of a polymeric insulation system.

In early experimental work into nanocomposite in connection with their dielectric properties, reduced space charge accumulation was reported in comparison with microcomposites [4]. This paper will evaluate the effects between of two main groups which are metallic oxides (zinc oxide) and rubber (polyacrylate) nanofillers. 


\section{SAMPLES PREPARATION}

Three type of samples were prepared which are different in their nano-sizes and content. The samples are unfilled XLPE, XLPE with a fraction of $1 \mathrm{wt} \%$ nano-metallic oxide and XLPE with a fraction of $1 \mathrm{wt} \%$ nanorubber. Table 1 shows the formation of samples. Additive free XLPE pellets were obtained from Borealis and the material name is Borlink LE4201R. There are 2 types of nanoparticles in this study which are Zinc Oxide (ZnO) nanoparticles were manufactured by US Research Nanomaterial and another nanoparticle is Polyacrylate (PA40) which was supplied by Kaneka. These nanoparticles are chosen based on the two main groups of the most common nanoparticles used for electrical insulating nanocomposite and simple chemical structure.

After the mixing process, all the nanocomposite materials were dried in an oven at 55C continuously for 16 hours respectively before the extrusion process. All the nanocomposite materials and XLPE pellets were compounded using an extruder at 130C. Then, sample films were prepared by hot-pressing under a pressure of $1000 \mathrm{psi}$ at $185 \mathrm{C}$ with an average thickness of $0.2 \mathrm{~mm}$ and a diameter of $70 \mathrm{~mm}$. Film samples were removed from the press were then cooled to room temperature.

Table 1. Sample used in the space charge measurement

\begin{tabular}{lc}
\hline Samples & Nanofiller Weight Percentage, wt $\%$ \\
\hline XLPE & 0 \\
XLPE+ZnO & 1 \\
XLPE+PA40 (Acrylic) & 1 \\
\hline
\end{tabular}

\section{EXPERIMENTAL WORKS}

The pulsed electro-acoustic (PEA) technique [5] was used to measure the space charge distribution for the various nanocomposites. The PEA principle is schematically represented in Fig. 1. When a pulse voltage is applied to the sample, it will produce an electric field impulse. Charges stored in the sample move under the effect of the Coulombs force. Acoustic waves are generated and propagated in the sample and then detected as electric signals by a piezoelectric sensor attached to the lower electrode. The amplitude of the signal is proportional to the charge quantity and the delay indicates the position of the charge, taking into account the distance from the sensor. A piezoelectric sensor transforms it into an electric signal whose amplitude is proportional to the local charge density. These signals are amplified and transferred to an oscilloscope [5].

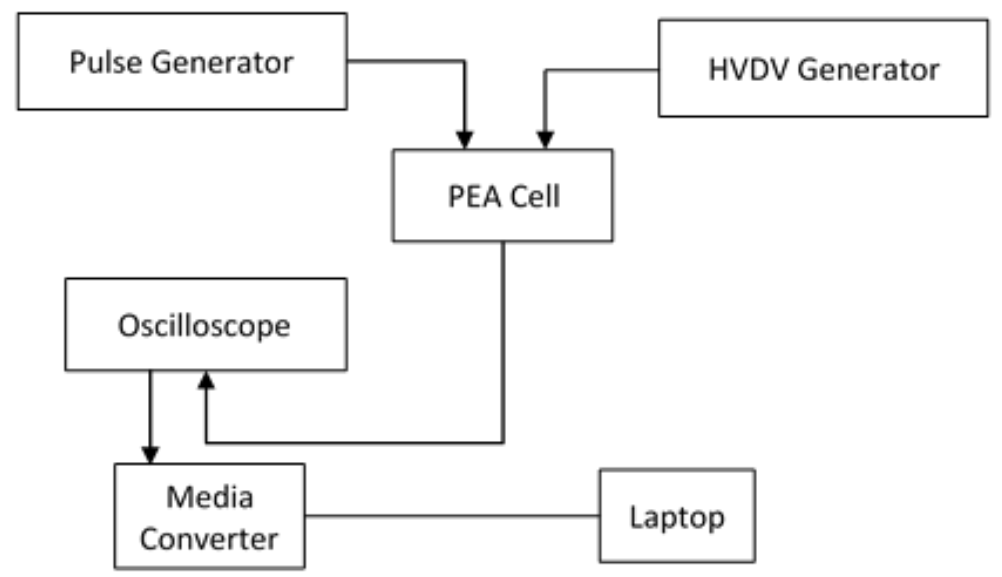

Figure 1. PEA cell scheme

Before performing the measurements, the electrodes, the samples, and the semiconductor overlaying the sample were thoroughly cleaned using isopropyl alcohol. In this study, a pulsed voltage of $1.8 \mathrm{~V}$ with a pulse length of $20 \mathrm{~ns}$ was applied into the sample for a few seconds to generate an acoustic signal wave. Then, calibration was conducted at low DC voltage of $1 \mathrm{kVmm}-1$ with short enough time of application to avoid space charge accumulation. The development of space charge was observed at three different DC voltage level which are 5, 10 and 15 
$\mathrm{kVmm}-1$ that lasts for 30 minutes at room temperature. The samples tested were the XLPE with nanoparticles for comparison in terms of space charge distribution while the pure XLPE material is used as reference. The dimension of sample and their cross section is illustrated in Fig. 2. In the present study, two different metallic electrode configurations were selected-brass and aluminium (Al).

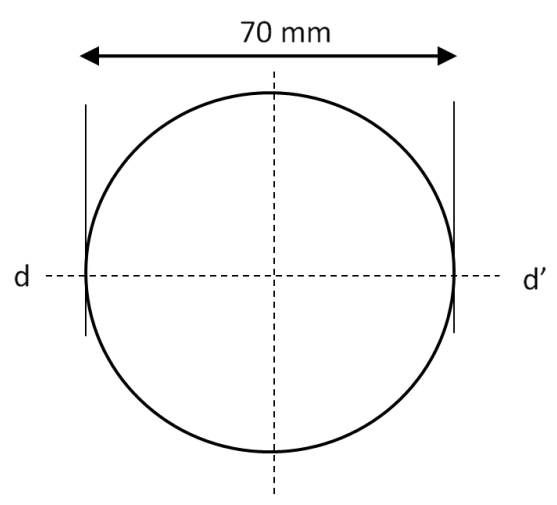

Figure 2. The dimension of sample

Then the sample was stressed at $1 \mathrm{kHz}$ for 30 minutes during which the time space charge was measured continuously. The reproducibility of space charge was validated by repeating the experiments three times for each group.

\section{EXPERIMENT RESULTS}

The space charge distributions were measured for 30 minutes after voltage was applied, inducing a short circuit between the electrodes. The two electrode positions are illustrated by the vertical dotted lines where the anode is located on the right while the cathode is on the left. In this experiment, there are two measurement results which were used to investigate the electrical properties of different nanofiller materials — space charge accumulation and electric field distribution.

\subsection{Accumulation of Space Charge}

As shown in Fig. 3, after dc voltage is applied, surface charge is induced on the electrode. When the voltage is more than $5 \mathrm{kV}$, hetero-charges which are the space charges within the insulation bulk begin to develop. Hetero-charge distribution was attributed by the cross-linking reaction and magnitude of space charge that rises with increasing magnitude of applied voltage. At $15 \mathrm{kV}$ applied voltage, the packets charges, both positive and negative charges propagate and meet in the middle. This leads to the beginning of recombination of charge.

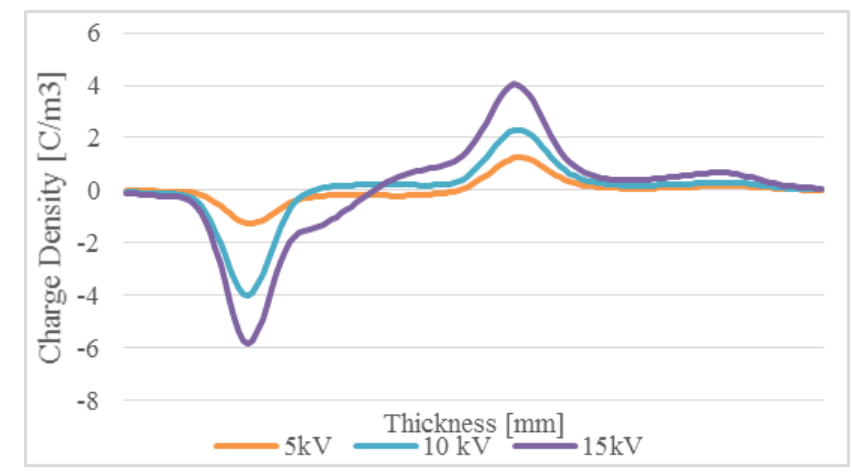

Figure 3. Unfilled XLPE

Figure 4 shows the space charge distribution in the XLPE with $1 \mathrm{wt} \%$ nanofillers after the voltage is applied. Although there is almost no obvious space charge, shown in Fig. 4a, but a very small amount of homocharge is evident near the cathode such that space charge development is minimal. It can be seen that the injected 


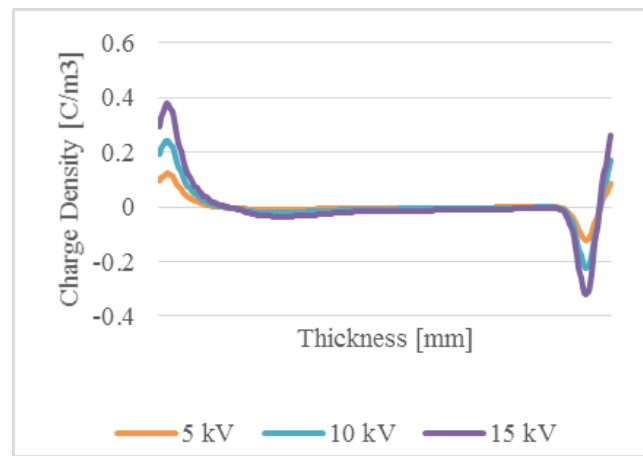

(a) ZLPE+ZnO

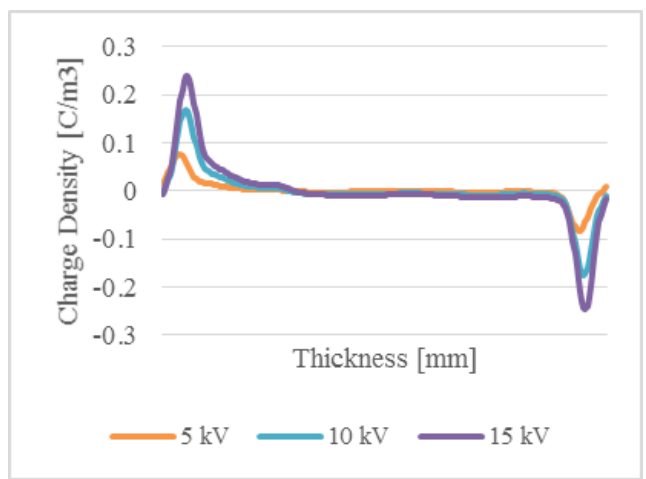

(b) XLPE+PA40 (Acrylic)

Figure 4. Space charge accumulation

carriers near the electrode forms a small amount of homo-charge and it increases with the duration of the applied voltage. Charges are dictated by homo-charges which means that the charge injection has the same polarity as the electrodes.

Figure $4 \mathrm{~b}$ shows a small amount of homo-charge is observed in XLPE with $1 \mathrm{wt} \%$ PA40 nanofillers. As shown, there is significant differences in charge profiles between XLPE with 1wt\% ZnO and XLPE with $1 \%$ PA40 nanofillers. The magnitude of charges in PA40 fillers is about 36.6\% less than that in ZnO fillers. From the charge profiles, it is clear that the space charge density increases with the increasing dc voltage. It is worth to note that the development of homo-charge is commonly associated with charge injection processes from the electrodes; that is, positive charge injection at the cathode and negative charge injection at the anode [6].

This trend seems to be consistent with the published results from [7],[8] which states the trend of the increasing magnitude of space charge indicates the possibility of the presence of nano filler may have delayed the charge injection or resulted in more homo-charges being generated due to the ionization process.

\subsection{Electric Field Distribution}

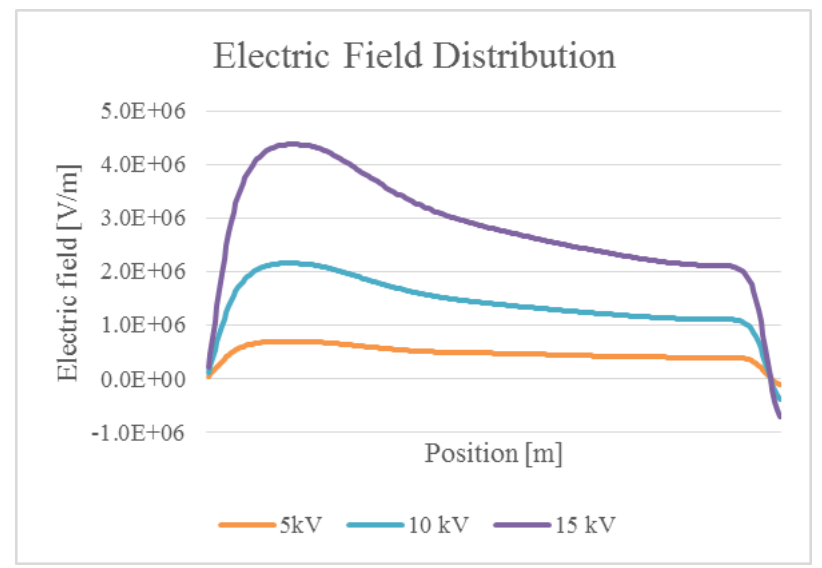

Figure 5. XLPE+ZnO

Figure 5 represents the electric field distribution of XLPE with $\mathrm{ZnO}$ nanofiller sample with three levels of applied voltage, 5,10 , and $15 \mathrm{kV}$. In the beginning of voltage application, a small value of electric field is developed in the vicinity of the electrodes. However, when $10 \mathrm{kV}$ is applied, the electric field begins to distort. At $15 \mathrm{kV}$, electric field distortion was increased to $99.35 \%$ larger than applied voltage.

Figure 6 shows the electric field inside neat the samples as a function of time. As it is well known, the presence of space charge inside insulating materials modifies the local electric field. Through PEA measurements the electric field profiles inside the insulator can also be obtained along the thickness of the sample. 


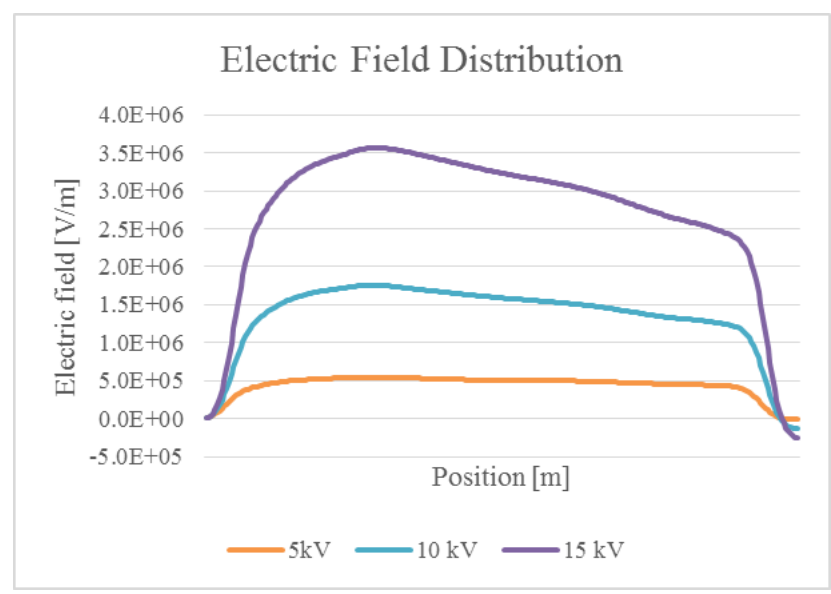

Figure 6. XLPE+PA40 (Acrylic)

In general, an increase in the applied voltage resulted in higher average space charge for most of the nanocomposite samples. It is observed that nanofillers demonstrated superior charge suppression properties [9]. The nanofillers introduced or increased the deep traps, leading to the decrease of charge carrier mobility. Additionally, nanofillers could also modify the surface charge transport resulting in the restriction of space charge accumulation.

\section{CONCLUSION}

It was observed that an increase in the applied voltage resulted in an increase in the amount of accumulated charge. This is attributed to enhanced charge injection when voltage is increased. Hetero-charges are formed for XLPE and these charges can be reduced by the addition of nanofillers. Homo-charges can be injected into nanocomposites with comparative ease. The observation of homo-charges in all the nanocomposites might be explained by enhanced charge injection due to the addition of nanofillers to XLPE. The samples reached fast charge saturation during polarization and fast depletion of charges during depolarization.

\section{ACKNOWLEDGEMENT}

The main author would like to express her appreciation to Universiti Teknikal Malaysia Melaka (UTeM) and Ministry of Education, Malaysia (MOE) for supporting her PhD work. This research is also supported by the cable diagnostic team from Tenaga Nasional Berhad (TNB) Research.

\section{REFERENCES}

[1] Y. M. Kim, Y. K. Cha, K. J. Lim, J. H. Nam, and G. J. Lee, "Electrical insulation evaluation of crosslinked polyethylene nanocomposite blended with ZnO," Proceedings of 2012 IEEE International Conference on Condition Monitoring and Diagnosis, CMD 2012, no. September, pp. 1242-1245, 2012.

[2] G. C. Montanari, "Bringing an insulation to failure: The role of space charge," IEEE Transactions on Dielectrics and Electrical Insulation, vol. 18, no. 2, pp. 339-364, 2011.

[3] K. Lau, A. Vaughan, G. Chen, I. Hosier, A. Holt, and K. Ching, "On the space charge and DC breakdown behavior of polyethylene/silica nanocomposites," IEEE Transactions on Dielectrics and Electrical Insulation, vol. 21, no. 1, pp. 340-351, 2014.

[4] Y. Wang, G. Chen, and A. Vaughan, "Space charge dynamics in silica-based polyethylene nanocomposites," 2014 IEEE Conference on Electrical Insulation and Dielectric Phenomena, CEIDP 2014, pp. 727-730, 2014.

[5] Y. Li, M. Yasuda, and T. Takada, "Pulsed Electroacoustic Method For Measurement Of Charge Accumulation In Solid Dielectrics," IEEE Transactions on Dielectrics and Electrical Insulation, vol. 1, no. 2, pp. 188-195, 1994.

[6] G. Chen, C. Zhang, and G. Stevens, "Space charge in LLDPE loaded with nanoparticles," in Annual Report Conference on Electrical Insulation and Dielectric Phenomena, CEIDP, 2007, pp. 275-278.

[7] J. Li, H. Xu, B. Du, and A. S. Preparation, "Space Charge Accumulation Characteristics in Ethylene-Vinyl Acetate Copolymer Filled with ZnO Nanoparticles," in IEEE International Conference on Dielectrics, 2016. 
[8] T. Arakane, T. Motchizuki, N. Adachi, H. Miyake, Y. Tanaka, Y. J. Kim, J. H. Nam, S. T. Ha, and G. J. Lee, "Space charge accumulation properties in XLPE with carbon nano-filler," in IEEE International Conference on Condition Monitoring and Diagnosis, 2012, pp. 328-331.

[9] K. Watanabe, A. Watanabe, and T. Okamoto, "Direct observation of time-dependent space charge profiles in XLPE cable under high electric fields," IEEE Transactions on Dielectrics and Electrical Insulation, vol. 1, no. 6, pp. 1068-1076, 1994.

\title{
BIOGRAPHIES OF AUTHORS
}

Anis Niza Ramani received her B. Eng. from Universiti Teknikal Malaysia Melaka in 2006 and
Master Degree from Universiti Teknologi Malaysia in 2009. She is currently a doctoral student in
the College of Engineering at Universiti Tenaga Nasional. Her research interests lie in dielectric
materials, high voltage engineering and space charge properties of insulating materials.

Anis Niza Ramani received her B. Eng. from Universiti Teknikal Malaysia Melaka in 2006 and the College of Engineering at Universiti Tenaga Nasional. Her research interests lie in dielectric materials, high voltage engineering and space charge properties of insulating materials.

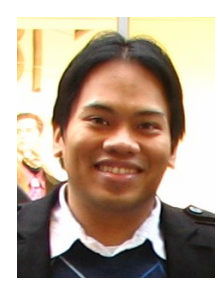

Azrul Mohd Ariffin is Associate Professor in the College of Engineering. He received the B.Eng. degree (2004) in electrical engineering and the Ph.D degree (2008) in electroluminescence phenomenon in insulating polymers from the University of Southampton, UK. He is now a Director of Programme Management Office (PMO) at Universiti Tenaga Nasional. His researches are in fields of high voltage engineering and electroluminescence phenomenon in insulating polymers. He is affiliated with IEEE and Board of Engineers, Malaysia (BEM) as a member.

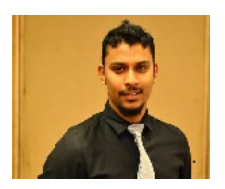

Gobinath Vijian is received B.Eng (Hons) degree in Electrical Power Engineering from University of Tenaga Nasional in 2016. Now he is currently pursuing post graduate studies in the same university. His current research interest include new insulation material to replace xlpe in underground cable that are widely used in Malaysias largest electricity provider, the TNB.

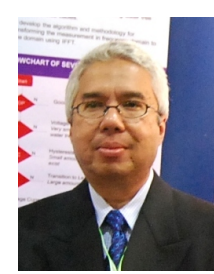

\begin{abstract}
Ahmad Basri Abd. Ghani is received his B.Eng (Hons) in Electrical Engineering degree from University of Southampton, UK in 1992. In 2002, he received his M.Eng in Engineering Management degree from University Tenaga Nasional, Malaysia, and in 2008 he received his PhD in Engineering degree from University Tenaga Nasional, Malaysia. In 1992, he joined Tenaga Nasional Berhad (TNB) as Operation \& Maintenance Engineer, and in year 2000, he joined TNB Research as the Principal Researcher. He is a registered Professional Engineer with Board of Engineer Malaysia since 1995. Since 2014, he has been a Senior Manager in TNB Research. His current research interests include underground cable, partial discharges, nanodielectrics, cable ampacity calculations, space charge \& high voltage technology.
\end{abstract}

